\title{
Ultrafast, all-optical regeneration functionalities inside a Kerr-nonlinear platform.
}

\author{
Gino Priem, Peter Vandersteegen, Peter Bienstman, Geert Morthier, and Roel Baets \\ Photonics Research Group, Dept. of Information Technology, Ghent University - IMEC, Belgium \\ Gino.Priem@intec.UGent.be
}

\begin{abstract}
We theoretically investigate the potential of a Kerrnonlinear resonator for all-optical regeneration. In combination with an optical amplifier, this ultra-compact structure allows for an extremely flexible approach in terms of bandwidth and regenerative strength, which can readily be integrated into future nonlinear platforms.
\end{abstract}

\section{INTRODUCTION}

Regeneration in an optical network is used for restoration of the optical signal from degradations due to noise and dispersion. In actual networks, this is mainly done by means of O-E-O (optical-electrical-optical) conversion. To reduce cost and to improve transparency, several all-optical configurations have already been proposed. Two important roads that are examined today are by means of nonlinear optical loop mirrors (NOLMs) [1] and by using optical amplifiers in a MachZehnder interferometer (MZI) [2]. In the first case, the incoming pulse is split into a co- and contrapropagating wave inside the NOLM and depending on the power of the pulse, a Kerrnonlinear phase shift arises between the co- and contrapropagating part, which leads to either destructive or constructive interference of both pulse parts at the output of the NOLM. Although this method is very robust, integration is difficult as NOLMs are typically constructed from several hundred meters of highly nonlinear fiber. In the second approach, a refractive index change between both MZI arms is caused by the optical amplifier and a differential mode of operation is used to eliminate the carrier lifetime limitations, leading to a very compact, low-power, but rather complex component.

In highly integrated all-optical platforms, losses and crosstalk between channels may require on-board regeneration. This may in particular be the case for future nonlinear platform - which may for example include ultrafast all-optical switching possibilities, memory operation, ... - in which power level and crosstalk handling is primordial. In such a case, it may be desirable to implement the regeneration capability on the nonlinear board itself to avoid additional integration steps. In Kerr-nonlinear systems, regeneration can straightforwardly be integrated by using the nonlinear reshaping potential of a single resonator structure.

In this paper, the reshaping function of a Kerr-nonlinear resonator structure is investigated and evaluated in terms of bandwidth and regenerative strength. It is shown that this function provides extremely versatile regenerative capabilities, which allow it to be optimized to specific circumstances.

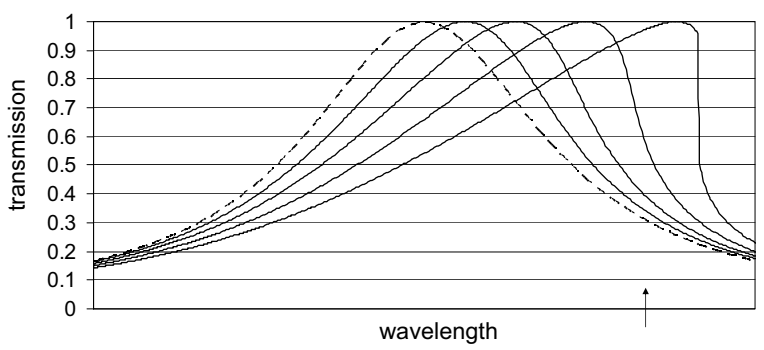

Fig. 1. Kerr-nonlinear shift of the resonator wavelength. The dotted line represents the linear transmission curve. Arbitrary units are used for the wavelength and power levels required for the nonlinear operation.

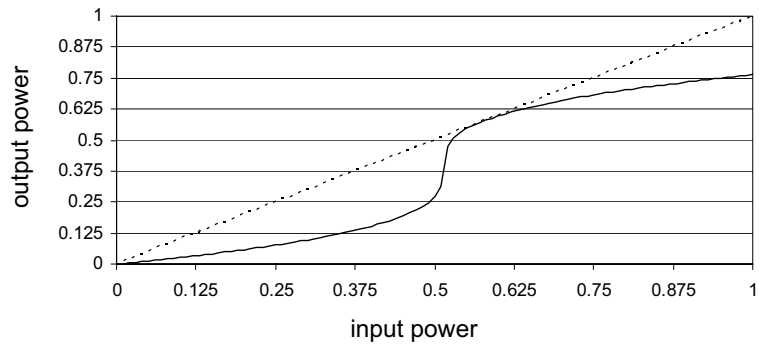

Fig. 2. Output - input power relation at the wavelength indicated by the arrow in Figure 1. Both axis are plotted in arbitrary units. A linear curve is plotted as a dotted line for reference.

\section{REGENERATION SCHEME.}

Inside a resonating structure such as a ring resonator, the optical Kerr effect induces a shift of the resonance wavelength which is proportional to the optical power inside the resonator cavity [3], [4] (Figure 1). This shift gives rise to an asymmetry in the transmission curve of the structure, which becomes more pronounced with the power level. As a result, an S-shaped output-input power curve can be obtained at the specific wavelength, as shown in Figure 2. Such a curve forms the basis for optical pulse reshaping.

By choice of the wavelength offset (with respect to the linear resonance wavelength), the level of reshaping can be optimized: the larger the offset, the larger the regenerative capability. Data rates that can be handled in this way, are only limited by the resonator bandwidth, as the response time of the Kerr effect is of the order of $f s$, and can therefore be adjusted by design. 
To also overcome losses in the system, an additional optical amplifier is placed in front of the resonator. This amplifier is used for both signal re-amplification and additionally for signal pre-conditioning to trigger the reshape function of the Kerr-nonlinear resonator.

\section{EXAMPLE}

Consider a $40 \mathrm{~Gb} / \mathrm{s}$ data signal centered around a wavelength of $\lambda=1.55 \mu \mathrm{m}$ that is specified by an average power of $P_{a v}=0.1 \mathrm{~mW}$ and extinction ratio $\frac{P_{1, i n}}{P_{0, i n}}=2$. The noise on the signal is assumed to be neglegible. However, when passing through the system, this signal will accumulate additional noise characterized by a rms value of $0.007 \mathrm{~mW}$. This corresponds to a bit error rate of $10^{-6}$ [5]. Suppose now that at the end of the system, a bit error rate of $B E R=10^{-12}$ would be required for a power of $P_{a v}=0.1 \mathrm{~mW}$. This is possible by first regenerating the signal before passing through the system. This is summarized in Figure 3, which shows the bit error rate as function of the received average power.

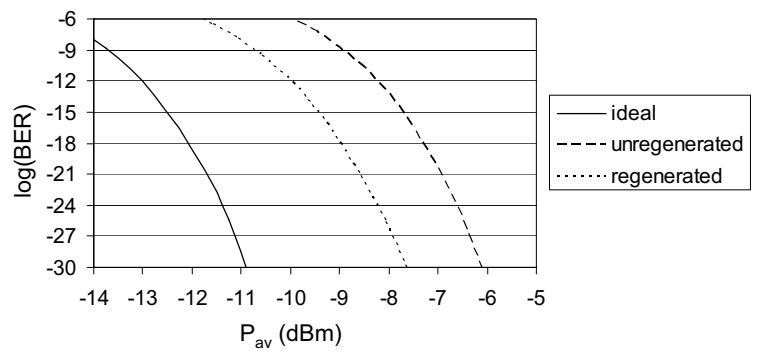

Fig. 3. Bit error rate of the data signal, considered in the text, at the end of the system as function of the received average power. In the unregenerated case, a bit error rate of only $10^{-6}$ is obtained for an average power of $P_{a v}=$ $0.1 \mathrm{~mW}$, while the design requires a value of $10^{-12}$. The bit error rate for an ideal input signal is shown as reference.

The reshaping function is assumed to be implemented in a $2 \mu \mathrm{m}$ radius, lossless ring resonator structure fabricated in AlGaAs [6]. The noise figure of the amplifier was assumed to be $N F=3$. To obtain the required bit error rate of $B E R=10^{-12}$, both resonator bandwidth, wavelength offset and amplifier gain were optimized by means of 1D analytical, nonlinear model [3], [4].

The following results were obtained: to obtain a bit error rate of $B E R=10^{-12}$, a minimal amplifier gain of $30.9 \mathrm{~dB}$ would be required, corresponding to an average power at the input of the ring resonator of $123 \mathrm{~mW}$. The optimum resonator bandwidth is $\Delta \lambda_{B W}=0.66 \mathrm{~nm}$ and the wavelength offset is $\Delta \lambda=0.51 \Delta \lambda_{B W}$. The obtained results were verified by means of 1D nonlinear FDTD simulations and are shown in Figures 4 and 5.

As can be seen, the improvement of the bit error rate in this case is clearly the result of extinction ratio reduction.

\section{CONCLUSION}

The potential of a Kerr-nonlinear resonator for reshaping has been investigated. In combination with an optical amplifier, this component provides an extremely flexible building block

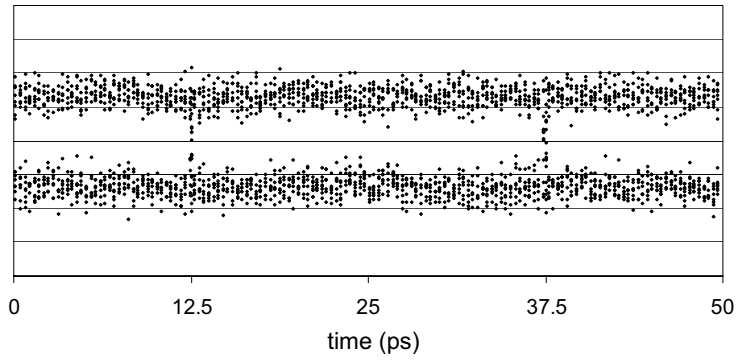

Fig. 4. Simulated eye-diagram in the unregenerated case. The average power level is $P_{a v}=0.1 \mathrm{~mW}$.

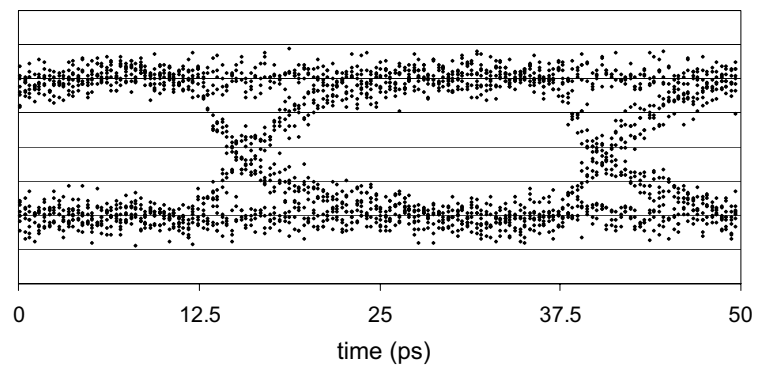

Fig. 5. Simulated eye-diagrams in the optimized regenerated case. The average power level is again $P_{a v}=0.1 \mathrm{~mW}$. Note the large extinction ratio improvement compared to the case of Figure 4.

for future nonlinear platforms. In terms of bandwidth, it is only limited by design, while its regenerative strength can be adjusted by the wavelength offset.

\section{ACKNOWLEDGMENT}

Part of this work has been performed in the context of the Belgian IAP PHOTON Network (IAP V/18).

Gino Priem and Peter Bienstman acknowledge the Flemish Fund for Scientific Research (FWO-Vlaanderen) for financial support.

\section{REFERENCES}

[1] M. Meissner et al., "12 db of noise reduction by a nolm-based $2 \mathrm{r}$ regenerator," IEEE Photon. Technol. Lett., vol. 15, no. 9, pp. 1297-1299, 2003.

[2] Y. Ueno et al., "Ultrafast $168 \mathrm{ghz} 1.5 \mathrm{ps} 1 \mathrm{fj}$ symmetric-mach-zehnder-type all-optical semiconductor switch," Jpn. J. Appl. Phys., vol. 39, pp. L806L808, 2000.

[3] G. Priem et al., "Design of all-optical nonlinear functionalities based on resonators," IEEE J. Select. Topics Quantum Electron., vol. 10, no. 5, pp. 1070-1078, 2004.

[4] —_, "Resonator-based all-optical kerr-nonlinear phase shifting: Design and limitations," J. Appl. Phys., vol. 97, no. 2, 2005.

[5] J. Mork et al., "Analytical expression for the bit error rate of cascaded all-optical regenerators," IEEE Photon. Technol. Lett., vol. 15, no. 10, pp. 1479-1481, 2003.

[6] A. Villeneuve et al., "Nonlinear refractive-index and two photonabsorption near half the band gap in algaas," Appl. Phys. Lett., vol. 62, pp. 2465-2467, 1993. 